\title{
Existence of Center for Planar Differential Systems with Impulsive Perturbations
}

\author{
Dengguo Xu \\ Department of Mathematics, Chuxiong Normal University, Chuxiong, Yunnan 675000, China \\ Correspondence should be addressed to Dengguo Xu; dengguoxu@163.com
}

Received 4 March 2015; Accepted 4 April 2015

Academic Editor: Ming Mei

Copyright (C) 2015 Dengguo Xu. This is an open access article distributed under the Creative Commons Attribution License, which permits unrestricted use, distribution, and reproduction in any medium, provided the original work is properly cited.

\begin{abstract}
We present a method that uses successor functions in ordinary differential systems to address the "center-focus" problem of a class of planar systems that have an impulsive perturbation. By deriving solution formulae for impulsive systems, several interesting criteria for distinguishing between the center and the focus of linear and nonlinear planar systems with state-dependent impulsions are established. The conditions describing the stability of the focus of the considered models are also given. The computing methods presented here are more convenient for determining the center of impulsive systems than those in the literature. Numerical examples are given to show the effectiveness of the theoretical results.
\end{abstract}

\section{Introduction}

Many differential evolutionary processes that arise in physical, chemical, and biological phenomena are characterized by the fact that they experience an abrupt change of state at certain moments in time. These processes can be modeled using impulsive differential equations. It is known, for example, that many phenomena involve thresholds and exhibit impulsive attributes, including bursting rhythm models (medicine and biology), optimal control models (economics), pharmacokinetics, and frequency modulated systems [1-3]. The theory of impulsive differential systems is much richer than the corresponding theory of systems without impulsive attributes. In general, there are three kinds of impulsive differential systems: (i) systems with impulses at fixed intervals, (ii) systems containing impulses with variable timing, and (iii) autonomously impulsive systems. Over the past few decades, the majority of research has been concentrated on systems with impulses at fixed intervals [4-6], while the other two kinds of impulsive differential systems were relatively less studied due to the difficulties caused by state-dependent impulsion. However, there have been significant developments in the theory of determining the center-focus for differential dynamical systems without impulsive attributes; the reader can refer to the monographs $[7,8]$. The center problem consists of distinguishing between a center and a focus at the origin of the system. In the case of a focus, the related problem is to analyze its stability and determine its highest possible order. Since the time of Poincaré [9], who initially defined the notion of the center for a real system of differential equations in the plane, the center-focus problem has been further extended by Liapunov [10], Bendixson [11], and Frommer [12]. Over the past couple decades, various kinds of techniques and algorithms have been developed, and extensive analyses have been consequently produced. Some results about center-focus problem for polynomial differential systems are presented in [13-16]. About the topic of discontinuous systems, there are also a lot of literatures; see, for example, [17-19].

There are many results regarding the bifurcation of impulsive differential systems, for example, [20-22]. However, to the best of our knowledge, the topic of the center-focus for systems with state-dependent impulsion has received less attention. In [23], the center-focus problem and the Hopf bifurcation of dynamical systems with impulses at variable intervals were studied by constructing a Poincaré map. An extension of these results to three-dimensional impulsive differential systems was presented in [24]. However, according to existing techniques, it is not easy to distinguish between the center and the focus in dynamical systems with 
a state-dependent impulsion. Motivated by this apparent lack of insight, in this paper we devoted our attention to a class of planar impulsive differential systems using successor functions. Our aim is to derive conditions for the existence of the center and focus and to analyze the stability of the focus in dynamical systems with a state-dependent impulsion. At the same time, a computational process will be presented. Due to the complexity caused by an impulse-jump in the systems, it is a challenge to obtain the solution of nonlinear impulsive systems using impulsive jumps of series solutions. In the case of linear impulsive systems, we extend the results in [23] to a more generalized scenario, and a convenient center-focus criterion is given. In the case of nonlinear impulsive systems, we propose a relatively simple method for distinguishing between the center and the focus of the systems, which is completely different from those in the existing literature.

The rest of this paper is organized as follows. In Section 2, the impulsive differential systems are formulated, and new definitions for the center and the focus for systems with a state-dependent impulsion are presented. The criteria for distinguishing between the center and the focus in linear and nonlinear impulsive systems are established by extension via the use of successor functions in Sections 3 and 4, respectively. Two numerical examples are given to demonstrate the effectiveness of the derived results in Section 5. Finally, our conclusions are drawn in Section 6.

The following conventions will be used throughout this paper. The transpose of matrix $M$ will be denoted by $M^{T}$ and the set of positive integer numbers by $N^{+}$. The symbols $\operatorname{sqrt}(x)$ and $\exp (x)$ denote $\sqrt{x}$ and $e^{x}$, respectively.

\section{Preliminaries}

Consider the following planar differential systems with an impulsive perturbation:

$$
\begin{aligned}
\dot{x} & =f(x), \quad x \notin T_{0}, \\
\Delta x & =B x, \quad x \in T_{0},
\end{aligned}
$$

where $x=\left[x_{1}, x_{2}\right]^{T} \in R^{2} ; B$ is a known $2 \times 2$ constant matrix; $f(x): R^{2} \rightarrow R^{2}$ is a real analytical vector function in the neighborhood of the origin such that $f(0)=0$; and $T_{0}$ is a subset of $R^{2}$. The phase point of (1) moves between two consecutive intersections with the set $T_{0}$ along the trajectories of (1). When the solution to (1) meets the set $T_{0}$ at the moment $\tau$, the point $x(\tau)$ has a jump $\Delta x=B x$, and $x\left(\tau^{+}\right)=x(\tau)+B x$.

The following assumptions will be imposed throughout the paper.

$\left(H_{1}\right)$ Set $T_{0}=\bigcup_{i=1}^{p} s_{i}, p \in N^{+}$, where $s_{i}$ are half-lines starting at the origin in the planar coordinate.

$\left(H_{2}\right)$ The Jacobian matrix of $f(x)$ has a pair of conjugate complex roots at the origin. The roots are denoted as $\alpha \pm \beta j, \beta>0$, where $j$ is complex unit.

$\left(H_{3}\right)$ Set $B=\left[\begin{array}{ll}b_{11} & b_{12} \\ b_{21} & b_{22}\end{array}\right]$; we denote $s_{i}^{+}=[I+B] s_{i}, i=$ $1,2, \ldots, p$. Let $\theta_{i}$ and $\gamma_{i}$ be angles $s_{i}$ and $s_{i}^{+}$with respect to the positive half-line of the first coordinate axis, and $0<\theta_{1}<\gamma_{1}<\cdots<\theta_{p}<\gamma_{p}<2 \pi$.
Next, one must make the transformation $x_{1}=r \cos \theta$, $x_{2}=r \sin \theta$. Then (1) is transformed, in polar coordinates, to the following differential system that possesses impulsive attributes:

$$
\begin{aligned}
\dot{r}(t) & =\alpha r+r R(r, \theta), \\
\dot{\theta}(t) & =\beta+Q(r, \theta) \\
\theta \neq \theta_{i}, & \\
\Delta r & =k(\theta) r, \\
\Delta \theta & =\Phi(\theta) \quad \\
& \theta=\theta_{i},
\end{aligned}
$$

where $R(r, \theta)$ and $Q(r, \theta)$ are at least first-order term in $r$ with coefficients depending on $\sin \theta$ or $\cos \theta$. When $\theta=\theta_{i}, i=$ $1,2, \ldots, p$, the point $\left(\theta_{i}, r_{i}\right)$ has jumps $\Delta \theta_{i}=\theta_{i}^{+}-\theta_{i}$ and $\Delta r_{i}=$ $r_{i}^{+}-r_{i} ;$ and

$$
\begin{gathered}
k(\theta)=\left[\left(\cos \theta+b_{11} \cos \theta+b_{12} \sin \theta\right)^{2}\right. \\
\left.+\left(\sin \theta+b_{21} \cos \theta+b_{22} \sin \theta\right)^{2}\right]^{1 / 2}-1, \\
\Phi(\theta)=\arctan \frac{\left(1+b_{22}\right) \sin \theta+b_{21} \cos \theta}{b_{12} \sin \theta+\left(1+b_{11} \cos \theta\right)}-\theta .
\end{gathered}
$$

Similar to the concepts of the center and focus in the ordinary differential systems $[7,25,26]$, the definition of the center and focus for impulsive differential systems (1) is given as follows.

Definition 1. For any sufficiently small $r_{0}>0$, let $r\left(\theta, \theta_{0}, r_{0}\right)$ denote the solution to a system (2a) and (2b) which starts at the point $\left(\theta_{0}, r_{0}\right)$. Then the origin of system (1) is called the center if $r\left(2 \pi+\theta, \theta_{0}, r_{0}\right)=r\left(\theta, \theta_{0}, r_{0}\right)$ and the focus if $r\left(2 \pi+\theta, \theta_{0}, r_{0}\right) \neq r\left(\theta, \theta_{0}, r_{0}\right)$. Moreover, the origin is called an asymptotically stable focus of the system (1) if $r(2 \pi+$ $\left.\theta, \theta_{0}, r_{0}\right)<r\left(\theta, \theta_{0}, r_{0}\right)$ and an unstable focus of the system (1) if $r\left(2 \pi+\theta, \theta_{0}, r_{0}\right)>r\left(\theta, \theta_{0}, r_{0}\right)$.

\section{Linear Impulsive Differential Systems}

Consider the following linear impulsive differential system:

$$
\begin{aligned}
\dot{x} & =A x, \quad x \notin T_{0}, \\
\Delta x & =B x, \quad x \in T_{0},
\end{aligned}
$$

where $A=\left[\begin{array}{cc}\alpha & -\beta \\ \beta & \alpha\end{array}\right], \beta>0$, and $x=\left[x_{1}, x_{2}\right]^{T} . T_{0}$ and the matrix $B$ are the same as those in $\left(H_{1}\right)$ and $\left(H_{3}\right)$.

Using polar coordinates, $x_{1}=r \cos \theta, x_{2}=r \sin \theta$, the system (4) reduces to

$$
\begin{aligned}
\dot{r}(t)= & \alpha r, \\
\dot{\theta}(t)= & \beta \\
& \theta \neq \theta_{i},
\end{aligned}
$$




$$
\begin{array}{r}
\Delta r=k(\theta) r, \\
\Delta \theta=\Phi(\theta) \\
\theta=\theta_{i},
\end{array}
$$

where $k(\theta)$ and $\Phi(\theta)$ are the same as those in (2a) and (2b).

Theorem 2. Let $\theta_{p+1}=2 \pi+\theta_{0}$. For $\theta \in\left(\theta_{i-1}, \theta_{i}\right], i=$ $1,2, \ldots, p+1$, the solution to system (5a) and (5b) with an initial value of $\left(\theta_{0}, r_{0}\right)$ can be formulated as

$$
\begin{aligned}
r\left(\theta, \theta_{0}, r_{0}\right) & \\
= & r_{0} \exp \left(\frac{\alpha}{\beta}\left(\theta-\theta_{0}-\sum_{j=1}^{i-1} \Phi\left(\theta_{j}\right)\right)\right) \\
& +\sum_{l=1}^{i-1} \Delta r_{l} \exp \left(\frac{\alpha}{\beta}\left(\theta-\theta_{l}-\sum_{j=l}^{i-1} \Phi\left(\theta_{j}\right)\right)\right),
\end{aligned}
$$

where $\theta_{0}<\theta_{1}$ and

$$
\begin{aligned}
& \Delta r_{l}=r_{0} k\left(\theta_{l}\right) \\
& \cdot \prod_{i=1}^{l-1}\left(1+k\left(\theta_{i}\right)\right) \exp \left(\frac{\alpha}{\beta}\left(\theta_{l}-\theta_{0}-\sum_{i=1}^{l-1} \Phi\left(\theta_{i}\right)\right)\right) \\
& \quad l=1,2, \ldots, i-1 .
\end{aligned}
$$

Proof. When $i=1, \theta \in\left(\theta_{0}, \theta_{1}\right]$, the solution to system (5a) and (5b) is

$$
r\left(\theta, \theta_{0}, r_{0}\right)=r_{0} \exp (\alpha t), \quad \theta=\theta_{0}+\beta t
$$

It follows that

$$
r\left(\theta, \theta_{0}, r_{0}\right)=r_{0} \exp \left(\frac{\alpha}{\beta}\left(\theta-\theta_{0}\right)\right) .
$$

This solution intersects with $\theta=\theta_{1}$ at point $\left(\theta_{1}, r_{1}\right)$, where

$$
r_{1}=r\left(\theta_{1}, \theta_{0}, r_{0}\right)=r_{0} \exp \left(\frac{\alpha}{\beta}\left(\theta_{1}-\theta_{0}\right)\right) .
$$

From (5b), we have

$$
\begin{aligned}
\theta_{1}^{+} & =\theta_{1}+\Phi\left(\theta_{1}\right) \\
r_{1}^{+} & =r_{1}+\Delta r_{1}=r_{0} \exp \left(\frac{\alpha}{\beta}\left(\theta_{1}-\theta_{0}\right)\right)+\Delta r_{1}, \\
\Delta r_{1} & =k\left(\theta_{1}\right) r_{1}=r_{0} k\left(\theta_{1}\right) \exp \left(\frac{\alpha}{\beta}\left(\theta_{1}-\theta_{0}\right)\right) .
\end{aligned}
$$

So, when $i=2, \theta \in\left(\theta_{1}, \theta_{2}\right]$. The solution to system (5a) and (5b) is given by

$$
\begin{aligned}
r & \left(\theta, \theta_{0}, r_{0}\right) \\
& =r_{1}^{+} \exp (\alpha t) \\
& =\left(r_{0} \exp \left(\frac{\alpha}{\beta}\left(\theta_{1}-\theta_{0}\right)\right)+\Delta r_{1}\right) \exp (\alpha t), \\
\theta & =\theta_{1}^{+}+\beta t=\theta_{1}+\Phi\left(\theta_{1}\right)+\beta t,
\end{aligned}
$$

from which we obtain

$$
\begin{aligned}
r\left(\theta, \theta_{0}, r_{0}\right)= & r_{0} \exp \left(\frac{\alpha}{\beta}\left(\theta-\theta_{0}-\Phi\left(\theta_{1}\right)\right)\right) \\
& +\Delta r_{1} \exp \left(\frac{\alpha}{\beta}\left(\theta-\theta_{1}-\Phi\left(\theta_{1}\right)\right)\right),
\end{aligned}
$$

which implies that (6) holds when $i=1$ and $i=2$.

Now, suppose that (6) is true when $i=m$. Namely, as $\theta \in\left(\theta_{m-1}, \theta_{m}\right]$

$$
\begin{aligned}
& r\left(\theta, \theta_{0}, r_{0}\right) \\
& =r_{0} \exp \left(\frac{\alpha}{\beta}\left(\theta-\theta_{0}-\sum_{j=1}^{m-1} \Phi\left(\theta_{j}\right)\right)\right) \\
& \quad+\sum_{l=1}^{m-1} \Delta r_{l} \exp \left(\frac{\alpha}{\beta}\left(\theta-\theta_{l}-\sum_{j=l}^{m-1} \Phi\left(\theta_{j}\right)\right)\right) .
\end{aligned}
$$

This solution intersects with $\theta=\theta_{m}$ at point $\left(\theta_{m}, r_{m}\right)$, where

$$
\begin{aligned}
r_{m}= & r\left(\theta_{m}, \theta_{0}, r_{0}\right) \\
= & r_{0} \exp \left(\frac{\alpha}{\beta}\left(\theta_{m}-\theta_{0}-\sum_{j=1}^{m-1} \Phi\left(\theta_{j}\right)\right)\right) \\
& +\sum_{l=1}^{m-1} \Delta r_{l} \exp \left(\frac{\alpha}{\beta}\left(\theta_{m}-\theta_{l}-\sum_{j=l}^{m-1} \Phi\left(\theta_{j}\right)\right)\right) ;
\end{aligned}
$$

it gives that from $(5 b)$

$$
\begin{aligned}
& \theta_{m}^{+}=\theta_{m}+\Phi\left(\theta_{m}\right), \\
& r_{m}^{+}=r_{m}+\Delta r_{m} .
\end{aligned}
$$

We can conclude that when $\theta \in\left(\theta_{m}, \theta_{m+1}\right]$, the solution to system (5a) and (5b) is

$$
r\left(\theta, \theta_{0}, r_{0}\right)=r_{m}^{+} \exp (\alpha t), \quad \theta=\theta_{m}^{+}+\beta t
$$

it follows that

$$
\begin{aligned}
r\left(\theta, \theta_{0}, r_{0}\right) & \\
= & r_{m}^{+} \exp \left(\frac{\alpha}{\beta}\left(\theta-\theta_{m}^{+}\right)\right) \\
= & \left(r_{m}+\Delta r_{m}\right) \exp \left(\frac{\alpha}{\beta}\left(\theta-\theta_{m}-\Phi\left(\theta_{m}\right)\right)\right) \\
= & r_{0} \exp \left(\frac{\alpha}{\beta}\left(\theta-\theta_{0}-\sum_{j=1}^{m} \Phi\left(\theta_{j}\right)\right)\right) \\
& +\sum_{l=1}^{m} \Delta r_{l} \exp \left(\frac{\alpha}{\beta}\left(\theta-\theta_{l}-\sum_{j=l}^{m} \Phi\left(\theta_{j}\right)\right)\right),
\end{aligned}
$$


where

$$
\begin{aligned}
\Delta r_{l}=k\left(\theta_{l}\right) r_{l}=r_{0} k\left(\theta_{l}\right) \prod_{i=1}^{l-1}\left(1+k\left(\theta_{i}\right)\right) & \\
\cdot \exp \left(\frac{\alpha}{\beta}\left(\theta_{l}-\theta_{0}-\sum_{i=1}^{l-1} \Phi\left(\theta_{i}\right)\right)\right) & \\
& \quad l=1,2, \ldots, i-1 .
\end{aligned}
$$

This implies that (6) holds when $i=m+1$. Using the reasoning of mathematical induction, we can immediately conclude that (6) is true for any $\theta \in\left(\theta_{i-1}, \theta_{i}\right], i=1,2, \ldots, p+$ 1. The proof is complete.

According to Definition 1 and Theorem 2, the following theorem holds, and it can distinguish between the center and the focus of a linear impulsive differential system.

Theorem 3. Denote $r\left(\theta, \theta_{0}, r_{0}\right)$ as the solution to system (5a) and (5b) with an initial value $\left(\theta_{0}, r_{0}\right)$, and let $r_{p+1}=$ $r\left(\theta_{p+1}, \theta_{0}, r_{0}\right)$. Then the origin of system (4) is

(i) a center if $r_{p+1}=r_{0}$,

(ii) an asymptotically stable focus if $r_{p+1}<r_{0}$,

(iii) an unstable focus if $r_{p+1}>r_{0}$.

Remark 4. When $\alpha \neq 0$, the origin is only a focus of the differential part in system (4). However, according to Theorem 3 , the origin may become a center of the impulsive differential system (4) when $\alpha \neq 0$. Whether the focus is stable or not has no dependency on the sign of $\alpha$. Therefore, the conclusion in Theorem 3 is actually an extension of the theory of centers and foci in ordinary differential systems.

Remark 5. When $\theta_{0}=0$ and $\theta \in\left(\theta_{p}, \theta_{p+1}\right]$, the solution (6) yields

$$
\begin{aligned}
r\left(\theta, \theta_{0}, r_{0}\right) & \\
= & r_{0} \exp \left(\frac{\alpha}{\beta}\left(\theta-\sum_{j=1}^{p} \Phi\left(\theta_{j}\right)\right)\right) \\
& +\sum_{l=1}^{p} \Delta r_{l} \exp \left(\frac{\alpha}{\beta}\left(\theta-\theta_{l}-\sum_{j=l}^{p} \Delta \theta_{j}\right)\right) \\
= & r_{0} \exp \left(\frac{\alpha}{\beta}\left(\theta-\sum_{j=1}^{p} \Phi\left(\theta_{j}\right)\right)\right) \\
& +\sum_{l=1}^{p} r_{0} k\left(\theta_{l}\right) \prod_{i=1}^{l-1}\left(1+k\left(\theta_{i}\right)\right) \\
& \times \exp \left(\frac{\alpha}{\beta}\left(\theta_{l}-\sum_{i=1}^{l-1} \Phi\left(\theta_{i}\right)\right)\right) \\
& \times \exp \left(\frac{\alpha}{\beta}\left(\theta-\theta_{l}-\sum_{j=l}^{p} \Phi\left(\theta_{j}\right)\right)\right)
\end{aligned}
$$

$$
\begin{aligned}
= & r_{0} \exp \left(\frac{\alpha}{\beta}\left(\theta-\sum_{j=1}^{p} \Phi\left(\theta_{j}\right)\right)\right) \\
& +\sum_{l=1}^{p} r_{0} k\left(\theta_{l}\right) \prod_{i=1}^{l-1}\left(1+k\left(\theta_{i}\right)\right) \\
& \times \exp \left(\frac{\alpha}{\beta}\left(\theta-\sum_{j=1}^{p} \Phi\left(\theta_{j}\right)\right)\right) \\
= & r_{0} \exp \left(\frac{\alpha}{\beta}\left(\theta-\sum_{j=1}^{p} \Phi\left(\theta_{j}\right)\right)\right) \\
& \times\left(1+\sum_{l=1}^{p} k\left(\theta_{l}\right) \prod_{i=1}^{l-1}\left(1+k\left(\theta_{i}\right)\right)\right) \\
= & r_{0} \exp \left(\frac{\alpha}{\beta}\left(\theta-\sum_{j=1}^{p} \Phi\left(\theta_{j}\right)\right)\right) \prod_{i=1}^{l}\left(1+k\left(\theta_{i}\right)\right),
\end{aligned}
$$

which is the solution of the nonperturbed system in [23]. Therefore, Theorem 2 of this paper extends the existing results to a more generalized case.

\section{Nonlinear Impulsive Differential Systems}

In this section, successor functions are used to study the center-focus problem of a class of nonlinear impulsive differential systems. We will consider the following nonlinear impulsive differential systems:

$$
\begin{aligned}
\dot{x} & =A x+f(x), \quad x \notin T_{0}, \\
\Delta x & =B x, \quad x \in T_{0},
\end{aligned}
$$

where $A=\left[\begin{array}{cc}\alpha & -\beta \\ \beta & \alpha\end{array}\right], \beta>0 ; x=\left[x_{1}, x_{2}\right]^{T}$; and $f(x)=\left[\begin{array}{l}f_{1}\left(x_{1}, x_{2}\right) \\ f_{2}\left(x_{1}, x_{2}\right)\end{array}\right]$ is a real analytical vector function in the neighborhood of the origin such that $f(x)=o(\|x\|)$ and $f_{i}(0,0)=0, i=1,2 . T_{0}$ and $B$ are the same as in (4).

Let us subject (21) to the transformation $x_{1}=r \cos \theta, x_{2}=$ $r \sin \theta$. Then (21) is transformed to

$$
\begin{aligned}
\dot{r}(t) & =\alpha r+r R(r, \theta), \\
\dot{\theta}(t) & =\beta+Q(r, \theta) \\
& \quad \theta \neq \theta_{i}, \\
\Delta r & =k(\theta) r, \\
\Delta \theta & =\Phi(\theta) \\
& \theta=\theta_{i},
\end{aligned}
$$


where $k(\theta)$ and $\Phi(\theta)$ are the same as in (5a) and (5b). Next,

$$
\begin{aligned}
R(r, \theta)= & \frac{1}{r} f_{1}(r \cos \theta, r \sin \theta) \cos \theta \\
& +\frac{1}{r} f_{2}(r \cos \theta, r \sin \theta) \sin \theta \\
Q(r, \theta)= & \frac{1}{r} f_{2}(r \cos \theta, r \sin \theta) \cos \theta \\
& -\frac{1}{r} f_{1}(r \cos \theta, r \sin \theta) \sin \theta
\end{aligned}
$$

It is clear that (22a) yields the following form:

$$
\frac{d r}{d \theta}=\frac{\alpha r+r R(r, \theta)}{\beta+Q(r, \theta)}
$$

Using the Taylor expansion, (24) can be expressed as follows:

$$
\frac{d r}{d \theta}=R_{1}(\theta) r+R_{2}(\theta) r^{2}+R_{3}(\theta) r^{3}+\cdots
$$

where, as is shown in [25], $R_{1}(\theta)=\alpha / \beta$ and $R_{i}(\theta), i=$ $2,3, \ldots$, is a polynomial in $\sin \theta$ and $\cos \theta$. Taking into account the continuity of the solution with respect to its initial values and the fact that $r=0$ is a solution to system (25), the solution $r\left(\theta, \theta_{0}, r_{0}\right)$ which starts at point $\left(\theta_{0}, r_{0}\right)$ exists for $\theta \in\left(\theta_{0}, \theta_{1}\right]$ when $r=r_{0}$ is a sufficiently small constant. This solution is analytical in $r_{0}$; thus $r\left(\theta, \theta_{0}, r_{0}\right)$ follows the Taylor expansion about $r_{0}$ :

$$
r\left(\theta, \theta_{0}, r_{0}\right)=r_{11}(\theta) r_{0}+r_{12}(\theta) r_{0}^{2}+r_{13}(\theta) r_{0}^{3}+\cdots
$$

it follows from the initial condition $r\left(\theta_{0}, \theta_{0}, r_{0}\right)=r_{0}$ that

$$
\begin{aligned}
& r_{11}\left(\theta_{0}\right)=1, \\
& r_{12}\left(\theta_{0}\right)=r_{13}\left(\theta_{0}\right)=\cdots=0 .
\end{aligned}
$$

Thus we can conclude that when $\theta \in\left(\theta_{0}, \theta_{1}\right]$, the solution to a nonlinear impulsive differential system (22a) and (22b) that starts at point $\left(\theta_{0}, r_{0}\right)$ is given by $(26)$. The solution $r\left(\theta, \theta_{0}, r_{0}\right)$ intersects $\theta=\theta_{1}$ at point $\left(\theta_{1}, r_{1}\right)$, where

$$
r_{1}=r_{11}\left(\theta_{1}\right) r_{0}+r_{12}\left(\theta_{1}\right) r_{0}^{2}+r_{13}\left(\theta_{1}\right) r_{0}^{3}+\cdots
$$

From (22b), we have

$$
\begin{aligned}
& \theta_{1}^{+}=\theta_{1}+\Phi\left(\theta_{1}\right), \\
& r_{1}^{+}=r_{1}+\Delta r_{1}=\left(1+k\left(\theta_{1}\right)\right) r_{1},
\end{aligned}
$$

using the same reasoning as before, when $\theta \in\left(\theta_{1}, \theta_{2}\right]$, the solution to system (22a) and (22b), which starts at point $\left(\theta_{1}^{+}, r_{1}^{+}\right)$, can be expressed by the following Taylor series:

$$
\begin{aligned}
r\left(\theta, \theta_{0}, r_{0}\right)= & r_{21}(\theta) r_{1}^{+}+r_{22}(\theta)\left(r_{1}^{+}\right)^{2}+r_{23}(\theta)\left(r_{1}^{+}\right)^{3} \\
& +\cdots \\
= & r_{21}(\theta)\left(1+k\left(\theta_{1}\right)\right) r_{1} \\
& +r_{22}(\theta)\left(1+k\left(\theta_{1}\right)\right)^{2} r_{1}^{2} \\
& +r_{23}(\theta)\left(1+k\left(\theta_{1}\right)\right)^{3} r_{1}^{3}+\cdots \\
= & \sum_{i=1}^{\infty} r_{2 i}(\theta)\left(1+k\left(\theta_{1}\right)\right)^{i} r_{1}^{i}
\end{aligned}
$$

Using the initial condition $r\left(\theta_{1}^{+}, \theta_{0}, r_{0}\right)=r_{1}^{+}$, one finds that $r_{2 i}(\theta), i=1,2, \ldots$, satisfys the following conditions:

$$
\begin{aligned}
& r_{21}\left(\theta_{1}^{+}\right)=1, \\
& r_{22}\left(\theta_{1}^{+}\right)=r_{23}\left(\theta_{1}^{+}\right)=\cdots=0 .
\end{aligned}
$$

The solution (30) intersects with $\theta=\theta_{2}$ at point $\left(\theta_{2}, r_{2}\right)$, where

$$
r_{2}=\sum_{i=1}^{\infty} r_{2 i}\left(\theta_{2}\right)\left(1+k\left(\theta_{1}\right)\right)^{i} r_{1}^{i} .
$$

From (22b), we have

$$
\begin{aligned}
& r_{2}^{+}=r_{2}+\Delta r_{2}=\left(1+k\left(\theta_{2}\right)\right) r_{2}, \\
& \theta_{2}^{+}=\theta_{2}+\Delta \theta_{2} .
\end{aligned}
$$

When $\theta \in\left(\theta_{2}, \theta_{3}\right]$, the solution to system (22a) and (22b) starting at point $\left(\theta_{2}^{+}, r_{2}^{+}\right)$has the following form:

$$
\begin{aligned}
r\left(\theta, \theta_{0}, r_{0}\right)= & r_{31}(\theta) r_{2}^{+}+r_{32}(\theta)\left(r_{2}^{+}\right)^{2}+r_{33}(\theta)\left(r_{2}^{+}\right)^{3} \\
& +\cdots \\
= & r_{31}(\theta)\left(1+k\left(\theta_{2}\right)\right) r_{2} \\
& +r_{32}(\theta)\left(1+k\left(\theta_{2}\right)\right)^{2} r_{2}^{2} \\
& +r_{33}(\theta)\left(1+k\left(\theta_{2}\right)\right)^{3} r_{2}^{3}+\cdots \\
= & \sum_{i=1}^{\infty} r_{3 i}(\theta)\left(1+k\left(\theta_{2}\right)\right)^{i} r_{2}^{i} .
\end{aligned}
$$

And $r_{3 i}(\theta)(i=1,2, \ldots)$ satisfies the following conditions:

$$
\begin{aligned}
& r_{31}\left(\theta_{2}^{+}\right)=1, \\
& r_{32}\left(\theta_{2}^{+}\right)=r_{33}\left(\theta_{2}^{+}\right)=\cdots=0 .
\end{aligned}
$$

When reasoning via mathematical induction, we can immediately conclude that when $\theta \in\left(\theta_{j-1}, \theta_{j}\right], j=1,2, \ldots, p+1$, $\theta_{p+1}=2 \pi+\theta_{0}$, the solution to system (22a) and (22b) is given by

$$
r\left(\theta, \theta_{0}, r_{0}\right)=\sum_{i=1}^{\infty} r_{j i}(\theta)\left(1+k\left(\theta_{j-1}\right)\right)^{i} r_{j-1}^{i},
$$


where $r_{1}=\sum_{i=1}^{\infty} r_{1 i}\left(\theta_{1}\right) r_{0}^{i}$ and

$$
r_{j-1}=\sum_{i=1}^{\infty} r_{j-1, i}\left(\theta_{j-1}\right)\left(1+k\left(\theta_{j-2}\right)\right)^{i} r_{j-2}^{i},
$$

$$
j=3,4, \ldots
$$

And $r_{j i}(\theta), i=1,2, \ldots$, satisfies the following conditions:

$$
\begin{aligned}
& r_{j 1}\left(\theta_{j-1}^{+}\right)=1, \\
& r_{j 2}\left(\theta_{j-1}^{+}\right)=r_{j 3}\left(\theta_{j-1}^{+}\right)=\cdots=0 .
\end{aligned}
$$

When $\theta \in\left(\theta_{j-1}, \theta_{j}\right], j=1,2, \ldots, p+1$, substituting the solution $r\left(\theta, \theta_{0}, r_{0}\right)$ into (25) and equating coefficient of $r_{j-1}^{m}, m=1,2, \ldots$, on both sides in $(25), r_{j i}(\theta), i=1,2, \ldots$, can be obtained. This is deduced as follows.

Substituting (26) into (25) yields the following equation:

$$
\begin{aligned}
& r_{11}^{\prime}(\theta) r_{0}+r_{12}^{\prime}(\theta) r_{0}^{2}+r_{13}^{\prime}(\theta) r_{0}^{3}+\cdots \\
& =R_{1}(\theta)\left(r_{11}(\theta) r_{0}+r_{12}(\theta) r_{0}^{2}+\cdots\right) \\
& \quad+R_{2}(\theta)\left(r_{11}(\theta) r_{0}+r_{12}(\theta) r_{0}^{2}+\cdots\right)^{2} \\
& \quad+R_{3}(\theta)\left(r_{11}(\theta) r_{0}+r_{12}(\theta) r_{0}^{2}+\cdots\right)^{3}+\cdots .
\end{aligned}
$$

Equating the coefficient of $r_{0}^{m}, m=1,2, \ldots$, on both sides of (39), one obtains

$$
\begin{aligned}
& r_{11}^{\prime}(\theta)=R_{1}(\theta) r_{11}(\theta), \\
& r_{12}^{\prime}(\theta)=R_{1}(\theta) r_{12}(\theta)+R_{2}(\theta) r_{11}^{2}(\theta), \\
& r_{13}^{\prime}(\theta)=R_{1}(\theta) r_{13}(\theta)+2 R_{2}(\theta) r_{11}(\theta) r_{12}(\theta) \\
& \quad+R_{3}(\theta) r_{11}^{3}(\theta),
\end{aligned}
$$

By solving these ordinary differential equations with the initial conditions in (27), it follows that

$$
\begin{aligned}
& r_{11}(\theta)=\exp \left(\frac{\alpha}{\beta}\left(\theta-\theta_{0}\right)\right) \\
& r_{12}(\theta)=\exp \left(\frac{\alpha}{\beta} \theta\right) \int_{\theta_{0}}^{\theta} R_{2}(\theta) \exp \left(\frac{\alpha}{\beta}\left(\theta-2 \theta_{0}\right)\right) d \theta \\
& r_{13}(\theta)=\exp \left(\frac{\alpha}{\beta} \theta\right) \int_{\theta_{0}}^{\theta}\left[2 R_{2}(\theta) \exp \left(\frac{\alpha}{\beta}\left(\theta-3 \theta_{0}\right)\right)\right. \\
& \cdot \int_{\theta_{0}}^{\theta} R_{2}(\theta) \exp \left(\frac{\alpha}{\beta} \theta\right) d \theta+R_{3}(\theta) \\
& \left.\cdot \exp \left(\frac{\alpha}{\beta}\left(2 \theta-3 \theta_{0}\right)\right)\right] d \theta
\end{aligned}
$$

Substituting (30) into (25) yields

$$
\begin{aligned}
& r_{21}^{\prime}(\theta)\left(1+k\left(\theta_{1}\right)\right) r_{1}+r_{22}^{\prime}(\theta)\left(1+k\left(\theta_{1}\right)\right)^{2} r_{1}^{2} \\
& +r_{23}^{\prime}(\theta)\left(1+k\left(\theta_{1}\right)\right)^{3} r_{1}^{3}+\cdots=R_{1}(\theta) \\
& +\left[r_{21}(\theta)\left(1+k\left(\theta_{1}\right)\right) r_{1}\right. \\
& +r_{22}(\theta)\left(1+k\left(\theta_{1}\right)\right)^{2} r_{1}^{2}+r_{23}(\theta)\left(1+k\left(\theta_{1}\right)\right)^{3} r_{1}^{3} \\
& +\cdots]+R_{2}(\theta)\left[r_{21}(\theta)\left(1+k\left(\theta_{1}\right)\right) r_{1}\right. \\
& +r_{22}(\theta)\left(1+k\left(\theta_{1}\right)\right)^{2} r_{1}^{2}+r_{23}(\theta)\left(1+k\left(\theta_{1}\right)\right)^{3} r_{1}^{3} \\
& +\cdots]^{2}+R_{3}(\theta)\left[r_{21}(\theta)\left(1+k\left(\theta_{1}\right)\right) r_{1}\right. \\
& +r_{22}(\theta)\left(1+k\left(\theta_{1}\right)\right)^{2} r_{1}^{2}+r_{23}(\theta)\left(1+k\left(\theta_{1}\right)\right)^{3} r_{1}^{3} \\
& +\cdots]^{3}+\cdots
\end{aligned}
$$

Equating the coefficient of $r_{1}^{m}, m=1,2, \ldots$, on both sides in (42) and solving the ordinary differential equations using the initial conditions in (31), one obtains the following result, which is similar to that obtained in (41):

$$
\begin{aligned}
& r_{21}(\theta)=\exp \left(\frac{\alpha}{\beta}\left(\theta-\theta_{0}\right)\right) \\
& r_{22}(\theta)=\exp \left(\frac{\alpha}{\beta} \theta\right) \int_{\theta_{0}}^{\theta} R_{2}(\theta) \exp \left(\frac{\alpha}{\beta}\left(\theta-2 \theta_{0}\right)\right) d \theta \\
& r_{23}(\theta)=\exp \left(\frac{\alpha}{\beta} \theta\right) \int_{\theta_{0}}^{\theta}\left[2 R_{2}(\theta) \exp \left(\frac{\alpha}{\beta}\left(\theta-3 \theta_{0}\right)\right)\right. \\
& \cdot \int_{\theta_{0}}^{\theta} R_{2}(\theta) \exp \left(\frac{\alpha}{\beta} \theta\right) d \theta+R_{3}(\theta) \\
& \left.\cdot \exp \left(\frac{\alpha}{\beta}\left(2 \theta-3 \theta_{0}\right)\right)\right] d \theta
\end{aligned}
$$

When reasoning via mathematical induction, we can conclude that when $\theta \in\left(\theta_{j-1}, \theta_{j}\right], j=1,2, \ldots, p+1$,

$$
\begin{aligned}
& r_{j 1}(\theta)=\exp \left(\frac{\alpha}{\beta}\left(\theta-\theta_{0}\right)\right), \\
& r_{j 2}(\theta)=\exp \left(\frac{\alpha}{\beta} \theta\right) \int_{\theta_{0}}^{\theta} R_{2}(\theta) \exp \left(\frac{\alpha}{\beta}\left(\theta-2 \theta_{0}\right)\right) d \theta, \\
& r_{j 3}(\theta)=\exp \left(\frac{\alpha}{\beta} \theta\right) \int_{\theta_{0}}^{\theta}\left[2 R_{2}(\theta) \exp \left(\frac{\alpha}{\beta}\left(\theta-3 \theta_{0}\right)\right)\right.
\end{aligned}
$$




$$
\begin{aligned}
& \cdot \int_{\theta_{0}}^{\theta} R_{2}(\theta) \exp \left(\frac{\alpha}{\beta} \theta\right) d \theta \\
& \left.+R_{3}(\theta) \exp \left(\frac{\alpha}{\beta}\left(2 \theta-3 \theta_{0}\right)\right)\right] d \theta
\end{aligned}
$$

The following theorem yields the solution to system (22a) and (22b).

Theorem 6. Let $\theta_{p+1}=2 \pi+\theta_{0}$. For $\theta \in\left(\theta_{j-1}, \theta_{j}\right], j=$ $1,2, \ldots, p+1$, the solution to system (22a) and (22b), which starts at the point $\left(\theta_{0}, r_{0}\right)$, is given by

$$
r\left(\theta, \theta_{0}, r_{0}\right)=\sum_{i=1}^{\infty} r_{j i}(\theta)\left(1+k\left(\theta_{j-1}\right)\right)^{i} r_{j-1}^{i},
$$

where

$$
\begin{aligned}
& k\left(\theta_{0}\right)=0, \\
& r_{1}=\sum_{i=1}^{\infty} r_{1 i}\left(\theta_{1}\right) r_{0}^{i}, \\
& r_{j-1}=\sum_{i=1}^{\infty} r_{j-1, i}\left(\theta_{j-1}\right)\left(1+k\left(\theta_{j-2}\right)\right)^{i} r_{j-2}^{i}, \\
& j=3,4, \ldots, p+1 .
\end{aligned}
$$

We define the successor function as

$$
\rho\left(r_{0}\right)=r\left(2 \pi+\theta_{0}, \theta_{0}, r_{0}\right)-r_{0}
$$

Thus, according to Definition 1, the following theorem holds.

Theorem 7. Assume that $r_{0}$ is sufficiently small, and $r\left(\theta, \theta_{0}, r_{0}\right)$ is the solution to system (22a) and (22b) that starts at point $\left(\theta_{0}, r_{0}\right)$. The origin is the center of system (21) if $\rho\left(r_{0}\right)=0$. Otherwise, the origin is either an asymptotically stable focus of system (21) under the condition $\rho\left(r_{0}\right)<0$ or an unstable focus under the condition $\rho\left(r_{0}\right)>0$.

Remark 8. In order to obtain the solution to nonlinear impulsive differential systems, we use the impulsive jumps of the series solutions in differential systems. This method is fundamentally different from that in [23], and our method plays an important role in distinguishing between the center and the focus of impulsive differential systems.

\section{Numerical Examples}

In this section, two numerical examples are given to illustrate the theoretical results presented in the previous sections.
Example 9. Consider the following linear impulsive differential system:

$$
\begin{aligned}
& \dot{x}_{1}=0.1 x_{1}-x_{2}, \\
& \dot{x}_{2}=x_{1}+0.1 x_{2}
\end{aligned}
$$

$$
x \notin l,
$$$$
\Delta x_{1}=\left(\frac{\sqrt{3}}{2} b_{0}-1\right) x_{1}-\frac{1}{2} b_{0} x_{2} \text {, }
$$$$
\Delta x_{2}=\frac{1}{2} b_{0} x_{1}+\left(\frac{\sqrt{3}}{2} b_{0}-1\right) x_{2}
$$

$$
x \in l \text {, }
$$

where $x=\left[x_{1}, x_{2}\right]^{T}, b_{0}>0$ is a real parameter, and the line $l$ is given by equation $x_{2}=(\sqrt{3} / 3) x_{1}, x_{1}>0$. Therefore $\theta_{1}=$ $\pi / 6, \theta_{0}<\theta_{1}<2 \pi$ and $b_{11}=(\sqrt{3} / 2) b_{0}-1, b_{12}=-(1 / 2) b_{0}$, $b_{21}=(1 / 2) b_{0}$, and $b_{22}=(\sqrt{3} / 2) b_{0}-1$. Using polar coordinates, $x_{1}=r \cos \theta, x_{2}=r \sin \theta,(48 \mathrm{a})$ and $(48 \mathrm{~b})$ reduce to

$$
\begin{aligned}
\dot{r}(t)= & 0.1 r, \\
\dot{\theta}(t)= & 1, \\
& \theta \neq \theta_{i}, \\
\Delta r= & k(\theta) r, \\
\Delta \theta= & \Phi(\theta) \\
& \theta=\theta_{i},
\end{aligned}
$$

where

$$
\begin{aligned}
& k(\theta)=\left[\left(\cos \theta+b_{11} \cos \theta+b_{12} \sin \theta\right)^{2}\right. \\
& \left.+\left(\sin \theta+b_{21} \cos \theta+b_{22} \sin \theta\right)^{2}\right]^{1 / 2}-1=b_{0}-1, \\
& \Phi(\theta)=\arctan \frac{\left(1+b_{22}\right) \sin \theta+b_{21} \cos \theta}{b_{12} \sin \theta+\left(1+b_{11} \cos \theta\right)}-\theta \\
& =\arctan \frac{\sqrt{3} \sin \theta+\cos \theta}{-\sin \theta+\sqrt{3} \cos \theta}-\theta .
\end{aligned}
$$

When $\theta \in\left(\theta_{0}, \theta_{1}\right]$, the solution to (49a) and (49b), which starts at point $\left(\theta_{0}, r_{0}\right)$, is

$$
r\left(\theta, \theta_{0}, r_{0}\right)=r_{0} \exp (0.1 t), \quad \theta=\theta_{0}+t
$$

that is,

$$
r\left(\theta, \theta_{0}, r_{0}\right)=r_{0} \exp \left(0.1\left(\theta-\theta_{0}\right)\right)
$$

This solution intersects with $\theta=\theta_{1}$ at point $\left(\theta_{1}, r_{1}\right)$, where

$$
r_{1}=r_{0} \exp \left(0.1\left(\theta_{1}-\theta_{0}\right)\right) .
$$


Considering

$$
\begin{aligned}
\theta_{1}^{+} & =\theta_{1}+\Delta \theta_{1}=\frac{\pi}{3} \\
r_{1}^{+} & =r_{1}+\Delta r_{1}=\left(1+k\left(\theta_{1}\right)\right) r_{1} \\
& =r_{0} b_{0} \exp \left(0.1\left(\theta_{1}-\theta_{0}\right)\right)
\end{aligned}
$$

thus, when $\theta \in\left(\theta_{1}, 2 \pi\right)$, the solution to (49a) and (49b) is

$$
r\left(\theta, \theta_{0}, r_{0}\right)=r_{1}^{+} \exp (0.1 t), \quad \theta=\theta_{1}^{+}+t .
$$

Thus, when $\theta \in\left(\theta_{1}, 2 \pi\right]$, the solution, in polar coordinates, to system $(49 \mathrm{a})$ and $(49 \mathrm{~b})$ that starts at the point $\left(\theta_{0}, r_{0}\right)$ is given by

$$
\begin{aligned}
r\left(\theta, \theta_{0}, r_{0}\right) & =r_{1}^{+} \exp \left(0.1\left(\theta-\theta_{1}^{+}\right)\right) \\
& =r_{0} b_{0} e^{0.1 \theta-0.1 \theta_{0}-\pi / 60}
\end{aligned}
$$

When $\theta=2 \pi+\theta_{0}, r\left(\theta, \theta_{0}, r_{0}\right)=r_{0} b_{0} e^{11 \pi / 60}$. Thus, the following proposition follows.

Proposition 10. The origin is the center (focus) of (48a) and $(48 b)$ if $b_{0}=e^{-11 \pi / 60}\left(b_{0} \neq e^{-11 \pi / 60}\right)$. Moreover, the origin is $a(n)$ stable (unstable) focus of (48a) and (48b) if $b_{0}<$ $e^{-11 \pi / 60}\left(b_{0}>e^{-11 \pi / 60}\right)$.

Numerical simulations are presented in Figure 1. The initial states of system (48a) and (48b) are $x_{1}(0)=5, x_{2}(0)=$ 0 . Here, we find a center for $b_{0}=e^{-11 \pi / 60} \approx 0.5622$ and an unstable focus for $b_{0}=e^{-\pi / 12} \approx 0.7697$; see Figures 1 (a) and (b). For a stable focus, the control parameter is chosen as $b_{0}=e^{-\pi / 4} \approx 0.4559$; see Figure $1(\mathrm{c})$.

Example 11. Consider the following nonlinear impulsive differential system:

$$
\begin{aligned}
& \dot{x}_{1}=-x_{2}, \\
& \dot{x}_{2}=x_{1}+x_{1}^{2}-x_{2}^{2} \\
& x \notin l, \\
& \Delta x_{1}=\left(\frac{\sqrt{3}}{2} b_{0}-1\right) x_{1}-\frac{1}{2} b_{0} x_{2}, \\
& \Delta x_{2}=\frac{1}{2} b_{0} x_{1}+\left(\frac{\sqrt{3}}{2} b_{0}-1\right) x_{2} \\
& x \in l,
\end{aligned}
$$

where $x=\left[x_{1}, x_{2}\right]^{T}$ and the line $l$ is given by equation $x_{2}=(\sqrt{3} / 3) x_{1}, x_{1}>0$. Thus $\theta_{1}=\pi / 6,0=\theta_{0}<\theta_{1}<2 \pi$. Applying the transformation in polar coordinates, (57b) is just transformed as (49b) and (57a) yield

$$
\frac{d r}{d \theta}=\frac{r^{2} \sin \theta \cos 2 \theta}{1+r \cos \theta \cos 2 \theta}
$$

using the Taylor expansion, we derive the following differential equation:

$$
\begin{aligned}
\frac{d r}{d \theta}= & \sin \theta \cos 2 \theta r^{2}-\sin \theta \cos \theta \cos ^{2} 2 \theta r^{3} \\
& +\sin \theta \cos ^{2} \theta \cos ^{3} 2 \theta r^{4}+\cdots
\end{aligned}
$$

it gives that

$$
\begin{gathered}
R_{1}(\theta)=0, \\
R_{2}(\theta)=\sin \theta \cos 2 \theta, \\
R_{3}(\theta)=-\sin \theta \cos \theta \cos ^{2} 2 \theta, \\
R_{4}(\theta)=\sin \theta \cos ^{2} \theta \cos ^{3} 2 \theta,
\end{gathered}
$$

When $\theta \in\left[\theta_{0}, \theta_{1}\right]$, the solution to system (59), which starts at the point $\left(\theta_{0}, r_{0}\right)$, is

$$
r\left(\theta, \theta_{0}, r_{0}\right)=r_{11}(\theta) r_{0}+r_{12}(\theta) r_{0}^{2}+r_{13}(\theta) r_{0}^{3}+\cdots ;
$$

it follows from (41) that

$$
\begin{aligned}
& r_{11}(\theta)=1, \\
& r_{12}(\theta)=\frac{1}{2} \cos \theta-\frac{1}{6} \cos 3 \theta-\frac{1}{3}, \\
& r_{13}(\theta)=\frac{1}{18} \cos \theta\left(20 \cos ^{5} \theta-42 \cos ^{3} \theta+8 \cos ^{2} \theta\right. \\
& +27 \cos \theta-12)-\frac{1}{18}
\end{aligned}
$$

And we have

$$
\begin{aligned}
\theta_{1} & =\frac{\pi}{6}, \\
k\left(\theta_{1}\right) & =b_{0}-1, \\
\Delta \theta_{1} & =\frac{\pi}{6}, \\
\theta_{1}^{+} & =\frac{\pi}{3}, \\
r_{1}^{+} & =b_{0} r_{1} .
\end{aligned}
$$

According to Theorem 6, when $\theta \in\left(\theta_{1}, 2 \pi\right]$, the solution to system (59) with the impulsive perturbation (49b), which starts at the point $\left(\theta_{0}, r_{0}\right)$, is

$$
\begin{aligned}
& r\left(\theta, \theta_{0}, r_{0}\right)=\sum_{i=1}^{\infty} r_{2 i}(\theta) b_{0}^{i} r_{1}^{i}=r_{21}(\theta) b_{0} r_{1}+r_{22}(\theta) \\
& \cdot b_{0}^{2} r_{1}^{2}+r_{23}(\theta) b_{0}^{3} r_{1}^{3}+\cdots=r_{21}(\theta) \\
& \cdot b_{0}\left[r_{11}\left(\theta_{1}\right) r_{0}+r_{12}\left(\theta_{1}\right) r_{0}^{2}+r_{13}\left(\theta_{1}\right) r_{0}^{3}+\cdots\right]
\end{aligned}
$$




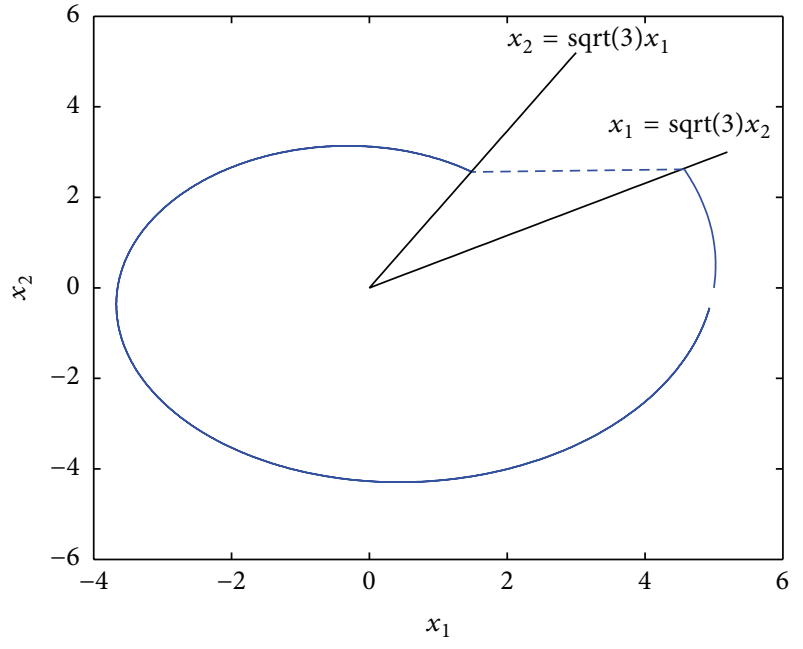

(a)

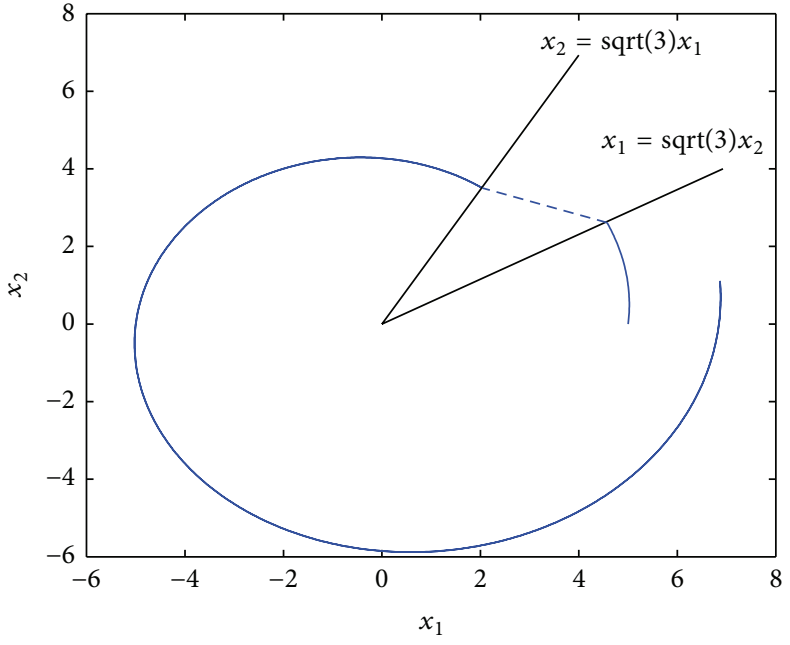

(b)

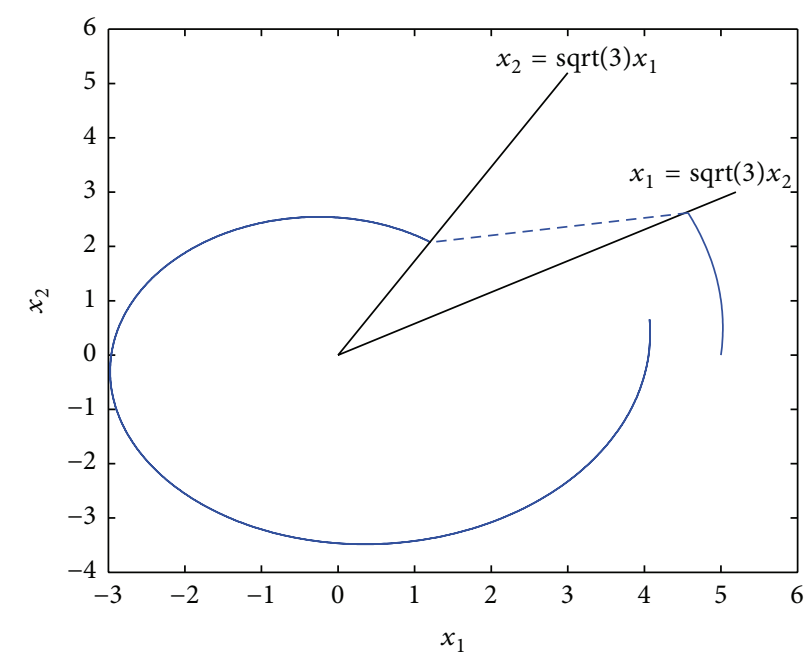

(c)

Figure 1: The center and focus of system (48a) and (48b). (a) The center, where $b_{0}=0.5622$. (b) An unstable focus, where $b_{0}=0.7697$. (c) A stable focus, where $b_{0}=0.4559$.

$$
\begin{aligned}
& +r_{22}(\theta) \\
& \cdot b_{0}^{2}\left[r_{11}\left(\theta_{1}\right) r_{0}+r_{12}\left(\theta_{1}\right) r_{0}^{2}+r_{13}\left(\theta_{1}\right) r_{0}^{3}+\cdots\right]^{2} \\
& +r_{23}(\theta) \\
& \cdot b_{0}^{3}\left[r_{11}\left(\theta_{1}\right) r_{0}+r_{12}\left(\theta_{1}\right) r_{0}^{2}+r_{13}\left(\theta_{1}\right) r_{0}^{3}+\cdots\right]^{3} \\
& +\cdots
\end{aligned}
$$

So, we have from (61) and (63) that

$$
\begin{aligned}
& r\left(2 \pi, 0, r_{0}\right)-r\left(0,0, r_{0}\right)=\left[r_{21}(2 \pi) b_{0} r_{11}\left(\theta_{1}\right)\right. \\
& \left.\quad-r_{11}(0)\right] r_{0}+\left[r_{21}(2 \pi) b_{0} r_{12}\left(\theta_{1}\right)\right. \\
& \left.\quad+r_{22}(2 \pi) b_{0}^{2} r_{11}^{2}\left(\theta_{1}\right)-r_{12}(0)\right] r_{0}^{2}
\end{aligned}
$$

$$
\begin{aligned}
& +\left[r_{21}(2 \pi) b_{0} r_{13}\left(\theta_{1}\right)+2 r_{22}(2 \pi) b_{0}^{2} r_{11}\left(\theta_{1}\right) r_{12}\left(\theta_{1}\right)\right. \\
& \left.+r_{23}(2 \pi) b_{0}^{3} r_{11}^{3}\left(\theta_{1}\right)-r_{13}(0)\right] r_{0}^{3}+\cdots=\left(b_{0}-1\right) r_{0} \\
& +\left(\frac{\sqrt{3}}{4}-\frac{1}{3}\right) b_{0} r_{0}^{2}+\left(\frac{9}{32}-\frac{\sqrt{3}}{6}-\frac{1}{18}\right) b_{0} r_{0}^{3}+\cdots
\end{aligned}
$$

Then, the following proposition follows.

Proposition 12. Assume that $r_{0}$ is sufficiently small. If $b_{0}>$ 1 , the origin is an unstable focus of system (57a) and (57b). If $b_{0}<1$, the origin is a stable focus of system (57a) and (57b). Otherwise, the coefficient of $r_{0}$ is zero and the coefficient of $r_{0}^{2}$ is positive in (65) when $b_{0}=1$; thus the origin is an unstable focus of system (57a) and (57b). 


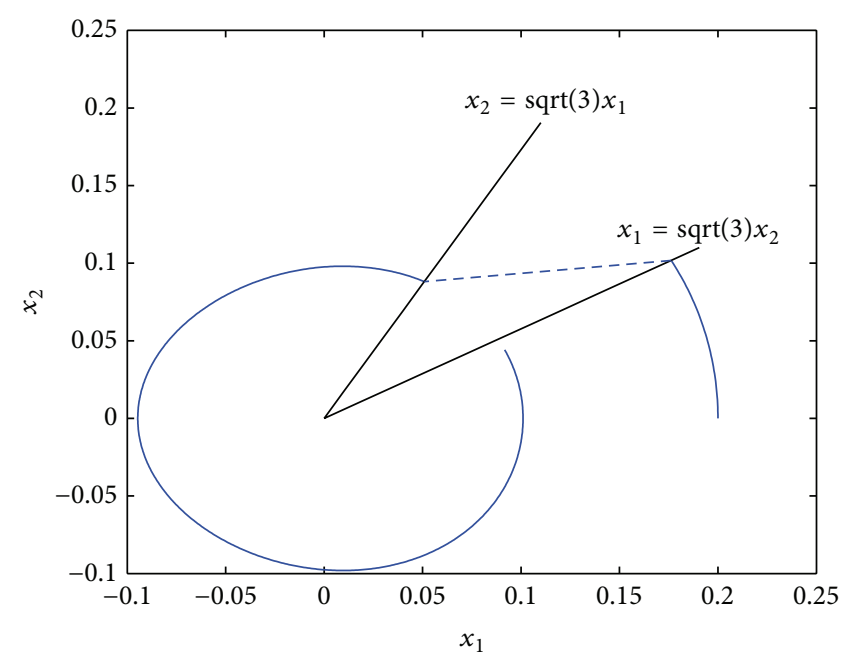

(a)

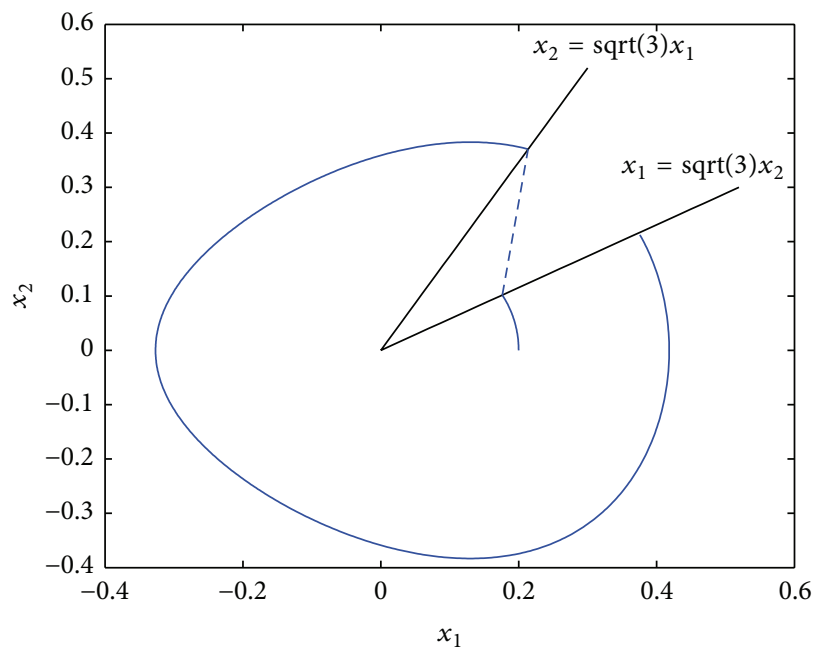

(b)

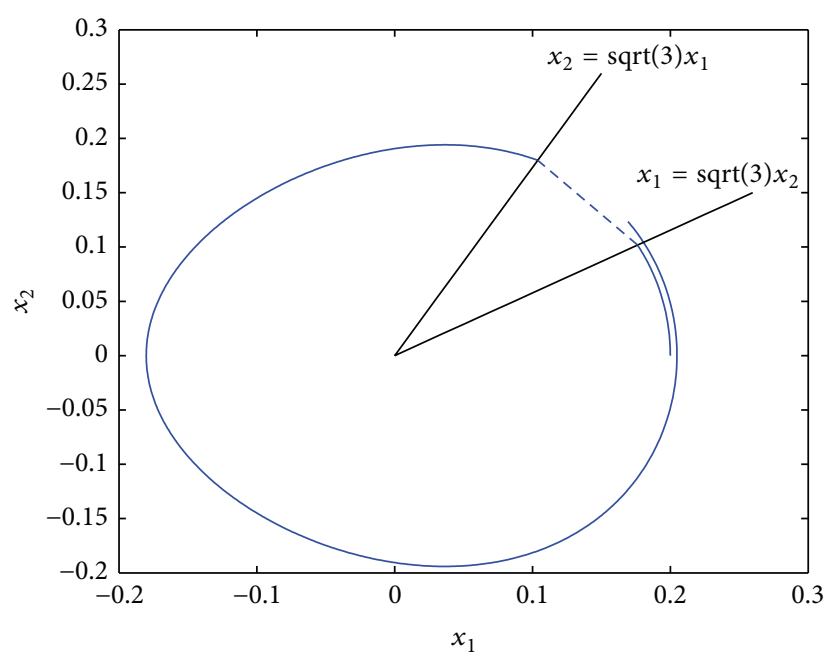

(c)

Figure 2: The focus of system (57a) and (57b). (a) A stable focus, where $b_{0}=0.5$. (b) An unstable focus, where $b_{0}=2.1$. (c) An unstable focus, where $b_{0}=1$.

Numerical simulations are displayed in Figure 2. The initial states of system (57a) and (57b) are $x_{1}(0)=0.2, x_{2}(0)=$ 0 . Here, we find a stable focus for $b_{0}=0.5$ and an unstable focus for $b_{0}=2.1$; see Figures $2(\mathrm{a})$ and 2(b). We find that the origin of the system (57a) and (57b) is still an unstable focus when $b_{0}=1$; see Figure 2(c).

\section{Conclusion}

In this paper, we studied the center-focus problem of planar differential systems that have a state-dependent impulsion. A criterion for distinguishing between the center and the focus in linear and nonlinear impulsive differential systems was established using successor functions. Our results are potentially useful if applied to the theory of impulsive differential systems. An extension of these results to threedimensional impulsive differential systems is an interesting topic that will be considered in future research.

\section{Conflict of Interests}

The author declares that there is no conflict of interests regarding the publication of this paper.

\section{Acknowledgments}

The author would like to thank the editors and reviewers for their helpful suggestions. This research is partially supported by the National Natural Science Foundation of China (61463002) and the Yunnan provincial Natural Science Foundation of China (2012FB175).

\section{References}

[1] V. Lakshmikantham, D. D. Bainov, and P. S. Simeonov, Theory of Impulsive Differential Equations, vol. 6 of Series in Modern Applied Mathematics, World Scientific Publishing, Singapore, 1989. 
[2] D. D. Bainov and P. S. Simeonov, Stability Theory of Differential Equations with Impulse Effects: Theory and Applications, Ellis Horwood, Chichester, UK, 1989.

[3] S. G. Pandit and S. G. Deo, Differential Systems Involving Impulses, Springer, New York, NY, USA, 1982.

[4] V. D. Milman and A. D. Myshkis, "On the stability of motion in the presence of impulses," Siberian Mathematical Journal, vol. 1, no. 2, pp. 233-237, 1960.

[5] J. Q. Lu, D. W. Ho, and J. D. Cao, "A unified synchronization criterion for impulsive dynamical networks," Automatica, vol. 46, no. 7, pp. 1215-1221, 2010.

[6] X. N. Liu and L. S. Chen, "Complex dynamics of holling type II lotka-volterra predator-prey system with impulsive perturbations on the predator," Chaos, Solitons and Fractals, vol. 16, no. 2, pp. 311-320, 2003.

[7] G. Sansone and R. Conti, Non-linear Differential Equations, Pergamon Press, New York, NY, USA, 1964.

[8] L. Perko, Differential Equations and Dynamical Systems, Springer, New York, NY, USA, 2000.

[9] H. Poincaré, "Mémoire sur les courbes définies par une équation différentielle (II)," Journal de Mathématiques Pures et Appliquées, vol. 8, pp. 251-296, 1882.

[10] M. A. Liapunov, Problème Général de la Stabilité du Mouvement, Princeton University Press, Princeton, NJ, USA, 1947.

[11] I. Bendixson, "Sur les courbes définies par des équations différentielles," Acta Mathematica, vol. 24, no. 1, pp. 1-88, 1901.

[12] M. Frommer, "Die integralkurven einer gewöhnlichen differentialgleichung erster ordnung in der umgebung rationaler unbestimmtheitsstellen," Mathematische Annalen, vol. 99, no. 1, pp. 222-272, 1928.

[13] D. M. Wang, "A class of cubic differential system with 6-tuple focus," Journal of Differential Equations, vol. 87, no. 2, pp. 305$315,1990$.

[14] L. S. Chen, Z. Y. Lu, and D. M. Wang, "A class of cubic systems with two centers or two foci," Journal of Mathematical Analysis and Applications, vol. 242, no. 2, pp. 154-163, 2000.

[15] Y. R. Liu and J. B. Li, "Bifurcations of limit cycles and center problem for a class of cubic nilpotent system," International Journal of Bifurcation and Chaos, vol. 20, no. 8, pp. 2579-2584, 2010.

[16] A. P. Sadovskii and T. V. Shcheglova, "Center conditions for a polynomial differential system," Differential Equations, vol. 49, no. 2, pp. 151-165, 2013.

[17] H. Giacomini, J. Giné, and J. Llibre, "The problem of distinguishing between a center and a focus for nilpotent and degenerate analytic systems," Journal of Differential Equations, vol. 227, no. 2, pp. 406-426, 2006.

[18] A. Gasull, J. Llibre, V. Mañosas, and F. Mañosas, "The focuscentre problem for a type of degenerate system," Nonlinearity, vol. 13, no. 3, pp. 699-729, 2000.

[19] A. Gasull and J. Torregrosa, "Center-focus problem for discontinuous planar differential equations," International Journal of Bifurcation and Chaos in Applied Sciences and Engineering, vol. 13, no. 7, pp. 1755-1765, 2003.

[20] L. Wang, L. Chen, and J. J. Nieto, "The dynamics of an epidemic model for pest control with impulsive effect," Nonlinear Analysis: Real World Applications, vol. 11, no. 3, pp. 1374-1386, 2010.

[21] L. N. Qian, Q. S. Lu, Q. G. Meng, and Z. S. Feng, "Dynamical behaviors of a prey-predator system with impulsive control," Journal of Mathematical Analysis and Applications, vol. 363, no. 1, pp. 345-356, 2010.
[22] G.-R. Jiang and Q.-G. Yang, "Periodic solutions and flip bifurcation in a linear impulsive system," Chinese Physics B, vol. 17, no. 11, pp. 4114-4122, 2008.

[23] M. U. Akhmet, "Perturbations and Hopf bifurcation of the planar discontinuous dynamical system," Nonlinear Analysis: Theory, Methods \& Applications, vol. 60, no. 1, pp. 163-178, 2005.

[24] M. U. Akhmet and M. Turan, "Bifurcation of three-dimensional discontinuous cycles," Nonlinear Analysis: Theory, Methods \& Applications, vol. 71, no. 12, pp. e2090-e2102, 2009.

[25] F. Dumortier, "Singularities of vector fields on the plane," Journal of Differential Equations, vol. 23, no. 1, pp. 53-106, 1977.

[26] A. D. Bruno, Local Methods in Nonlinear Differential Equations, Springer, Berlin, Germany, 1989. 


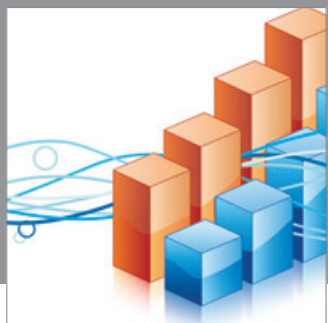

Advances in

Operations Research

mansans

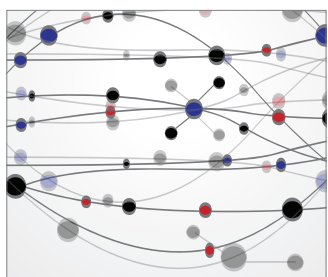

The Scientific World Journal
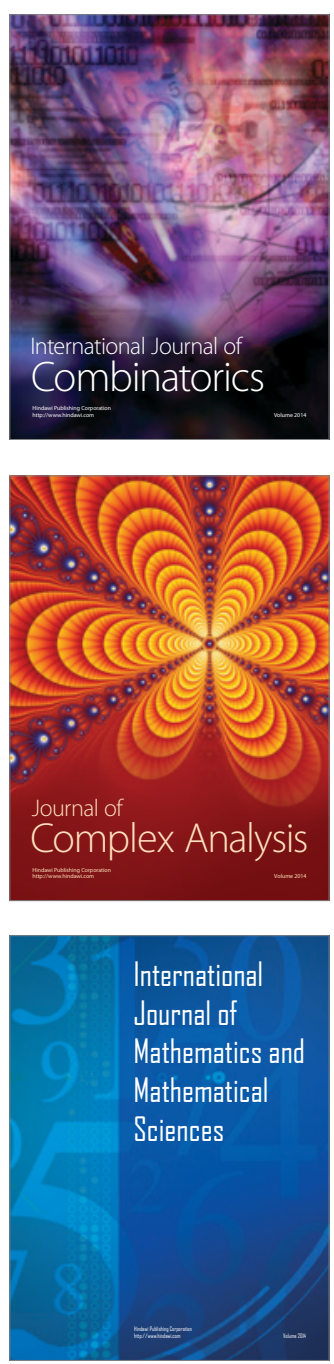
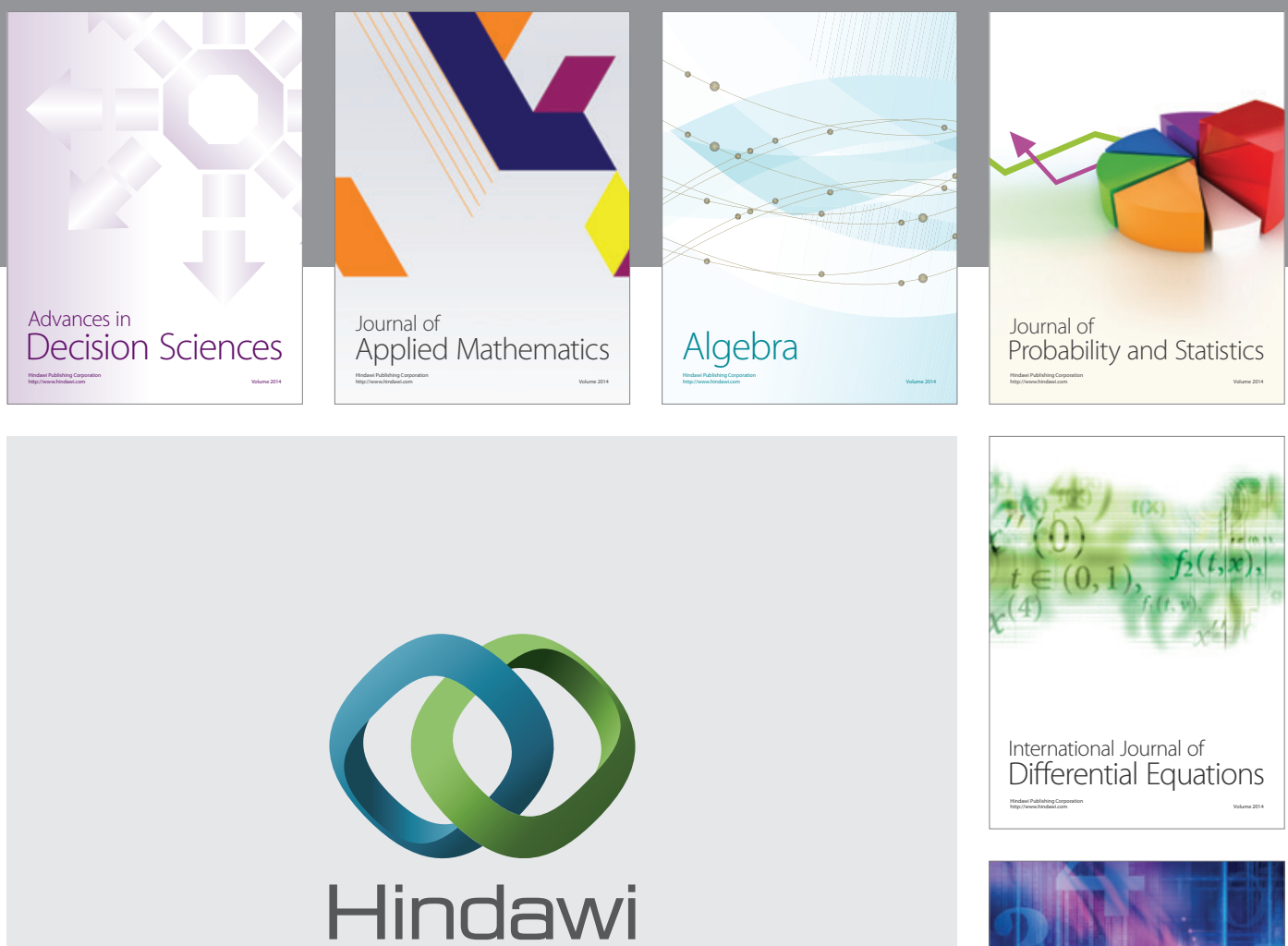

Submit your manuscripts at http://www.hindawi.com
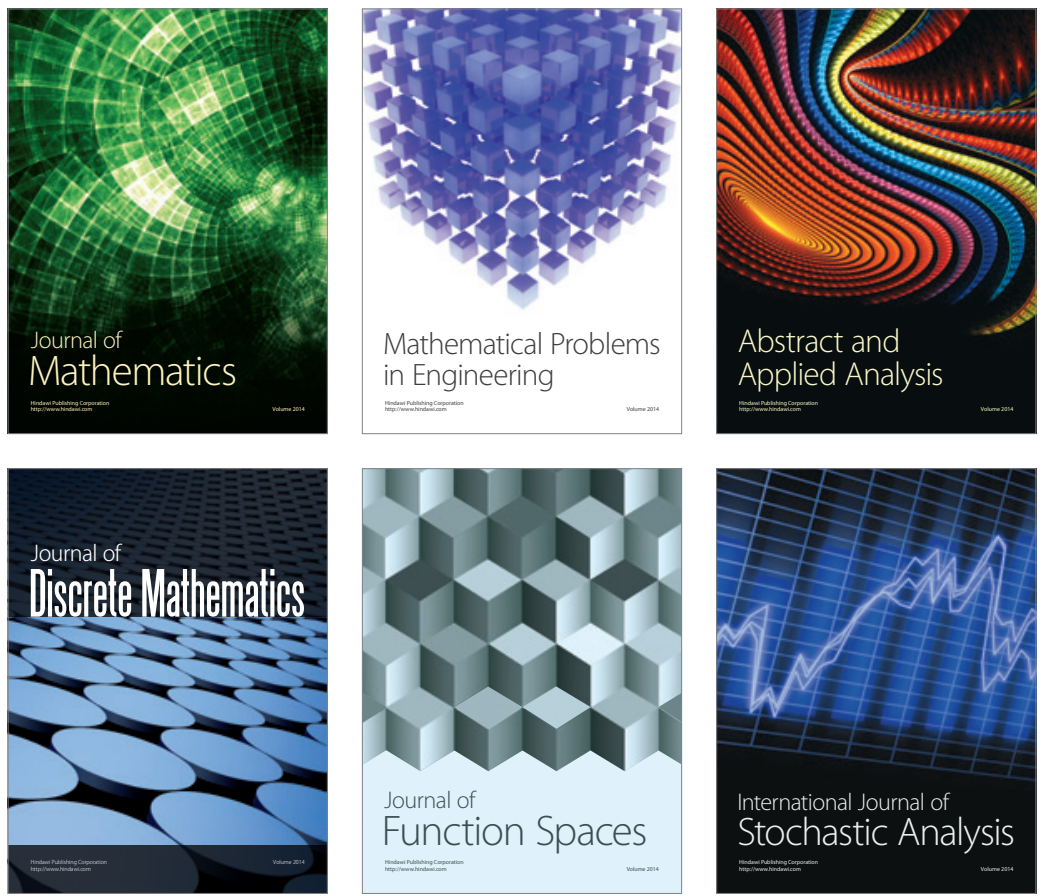

Journal of

Function Spaces

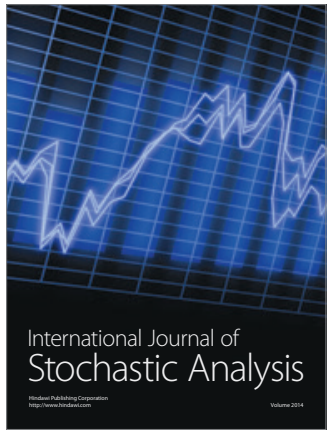

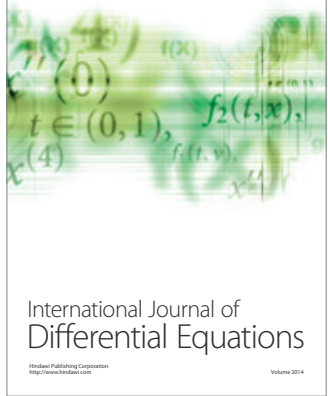
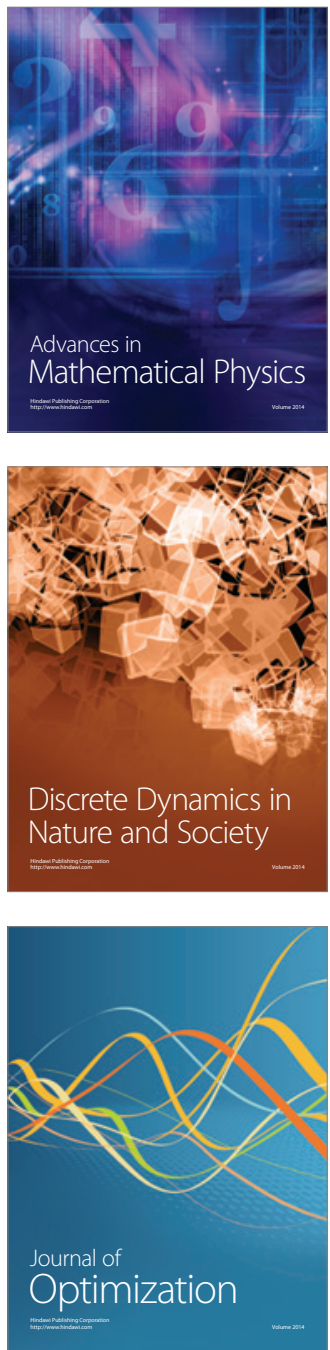